\title{
Synthesis of motion control of three-mass oscillating system based on solution of inverse dynamic problems
}

\author{
Kuznetsov N. K. \\ Head of the Department of Design and Standardization in \\ Mechanical Engineering \\ Irkutsk National Research Technical University \\ Irkutsk, Russia \\ knik@istu.edu \\ Dolgih E.S. \\ Department of Mine Machine and Electromechanical \\ System \\ Irkutsk National Research Technical University \\ Irkutsk, Russia
}

\author{
Iov I.A. \\ Engeneer \\ SLC-JH Ltd. \\ Irkutsk, Russia
}

Iov A.A.

Engeneer

SLC-JH Ltd.

Irkutsk, Russia

\begin{abstract}
In the article, using the example of a three-mass oscillating system, a procedure for the synthesis of motion control is presented, based on the determination, by solving the inverse dynamic problems, of the control actions that provide the desired character of the oscillating motions for individual partial systems. It is shown that the received control actions will be functions of time, which do not impose any restrictions on the structure of controllers, which can be realized by choosing the design parameters of the control object and by using closed automatic control systems. Since the feedback gains are determined directly from the mathematical model of the control object, the control system will have a weak sensitivity to parametric and coordinate perturbations. The results of numerical simulation of the effectiveness of the proposed method for limiting elastic oscillation are presented.
\end{abstract}

Keywords—control system, three-mass system, inverse problem, dynamic load, mechanical system.

\section{INTRODUCTION}

The actual problem of the creation of many technological and transport machines is the limitation of the level of oscillating movements of the actuating mechanisms in the transient operating modes. This problem is particularly important in the development of mining machines operating in intensive short-time modes, with frequent starts, breakdowns, reversals and latching actuating mechanism, and having elements with pronounced elastic properties (long shafts and cables). The current level of development of power converter technology and electric drive used in control systems of mining machines makes it possible to form with the required accuracy the prescribed laws of motion of the motor shaft in transient operation modes [1-3]. However, a further increase in the speed of movement associated with the need to increase the productivity of these machines will inevitably lead to the appearance of elastic oscillation and dynamic loads in the mechanical system and transmission of machines that reduce the speed and reliability of the operation of the actuator mechanism $[4,5]$.

The actuator mechanisms of many mining machines, which include digging excavator mechanisms (lifting hoist, traction, crowd, including gear and cable), mine hoisting machines one-end and two-terminal, which take into account the elasticity of the incoming and running-out branches of the cable on the head block and the moment inertia of the head block on the copra, are rather complex single-chain or branched multi-mass oscillating systems [6-8]. Depending on the research tasks, the mechanical system can be represented by a three- or four-mass system with variable parameters of elasticity and inertia of the actuator mechanism and the presence of backlashes in the gearbox.

To reduce dynamic loads and to limit the elastic oscillation of actuator mechanisms in transient operation modes, an active method of controlling oscillations is increasingly being used based on the use of standard electric drives of similar machines with feedbacks on the load in the elastic element [9-11]. The main problem in using this method of motion control is the problem of synthesis of the optimal structure and parameters of feedbacks under different operating conditions. In this connection, it is of particular interest to use the method of synthesis of control actions based on solving of an inverse dynamic problem by means of a mathematical model of the object by setting the desired character of the oscillational motions $[12,13]$. The received control actions will be functions of time, which do not impose any restrictions on the structure of controllers, and allow taking into account all parametric and coordinate perturbations. At the same time, the application of this approach to multi-mass oscillating systems, which are 
described by high-order interrelated differential equations, is a rather difficult task.

In this connection, it seems expedient to synthesize control actions by independently solving the inverse dynamic problems for individual partial systems of the control object, without taking into account their mutual connection.

In this article, using the example of a three-mass oscillating system, we describe the procedure for synthesizing control actions based on solving the inverse dynamic problems for individual partial systems.

\section{OBJECT AND METHOD OF INVESTIGATION}

The possibilities of the proposed approach will be considered using the example of a three-mass oscillating system, behavior the mechanical system is described by the following system of equations:

$$
\left\{\begin{array}{l}
J_{1} \ddot{\varphi}_{1}=M_{d v}-c_{12}\left(\varphi_{1}-\varphi_{2}\right)-b_{1} \dot{\varphi}_{1} \\
J_{2} \ddot{\varphi}_{2}=c_{12}\left(\varphi_{1}-\varphi_{2}\right)-c_{23}\left(\varphi_{2}-\varphi_{3}\right), \\
J_{3} \ddot{\varphi}_{3}=c_{23}\left(\varphi_{2}-\varphi_{3}\right)-M_{c}
\end{array}\right.
$$

where $\varphi_{1}$ - the coordinate of the movement of the first mass (drive); $\varphi_{2}$ - the coordinate of movement the intermediate mass (gearbox); $\varphi_{3}$ - the coordinate of the movement of the third mass (actuator mechanism); $J_{1}, J_{2}, J_{3}$ - reduced to drive inertia of the drive, gearbox and actuator mechanism; $M_{d v}$ and $M_{c}$ - the driving torque and the load torque; $c_{12}$ и $c_{23}$ - coefficients of stiffness of elastic elements; $b_{1}$ coefficient of viscous friction [13].

The structural scheme obtained on the basis of the system of equations (1) is shown in Fig. 1. As can be seen from this figure, there are cross-links between the individual partial subsystems 1 and 2. Therefore, a direct solution of the inverse dynamic problem, by expressing the elastic deviation of the last mass relative to the drive torque $M_{d v}$, is not possible.

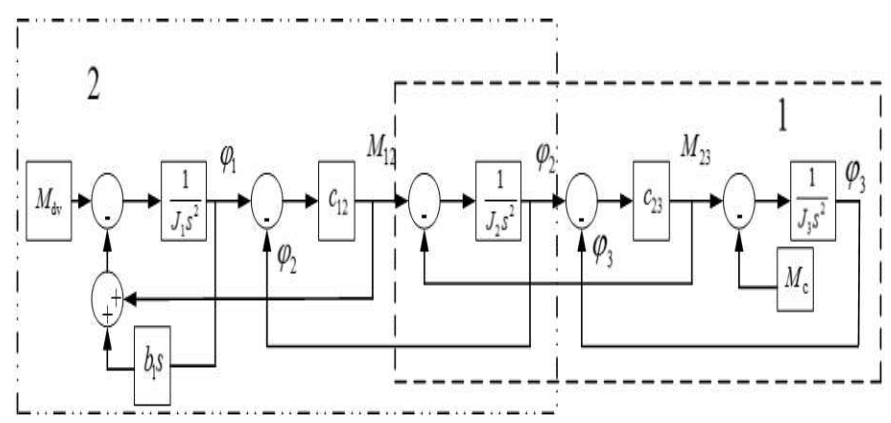

Fig. 1. Structural scheme of a three-mass mechanical system

Since the main task of control is to limit the oscillations of the latter mass, we first determine the required law of the change in the elastic torque $M_{12}$, at which a given character of the change in the elastic coordinate $\Delta \varphi_{23}$ is provided, using the last two equations of system (1) with load torque $M_{c}=0$.

$$
\Delta \ddot{\varphi}_{23}+\omega_{23}^{2} \Delta \varphi_{23}=\frac{M_{12}}{J_{2}},
$$

where $\Delta \varphi_{23}$ is the elastic deviation of the third mass; $M_{12}$ the torque transmitted through the elastic element with a stiffness coefficient $c_{12} ; \quad \omega_{23}=\sqrt{\frac{c_{23}\left(J_{2}+J_{3}\right)}{J_{2} J_{3}}}-$ partial frequency of oscillations of subsystem 1 .

We give the exponential law of the change in the coordinate $\Delta \varphi_{23}$ at which there are no oscillations:

$$
\Delta \varphi_{23}=C_{1} e^{\lambda_{1} t}+C_{2} e^{\lambda_{2} t},
$$

where $C_{1}, C_{2}$ are integration constants; $\lambda_{1}$ and $\lambda_{2}$ are different real or complex-conjugate numbers, such, that $\operatorname{Re} \lambda_{i}<0$.

Substituting coordinate (3) and its second derivative in equation (2), we find the dependence the required law of change of the elastic torque $M_{12}$ as a function of time:

$$
M_{12}=J_{2}\left(\lambda_{1}^{2}+\omega_{23}^{2}\right) C_{1} e^{\lambda_{1} t}+J_{2}\left(\lambda_{2}^{2}+\omega_{23}^{2}\right) C_{2} e^{\lambda_{2} t} .
$$

Expressing, using (3), the exponential dependences $C_{1} e^{\lambda_{1} t}$ and $C_{2} e^{\lambda_{2} t}$ through the coordinate $\Delta \varphi_{23}$ and its derivative under the initial conditions $\Delta \varphi_{23}(0)=\Delta \varphi_{23}, \Delta \dot{\varphi}_{23}(0)=\Delta \dot{\varphi}_{23}$, and substituting them in (4), we obtain the following relationship:

$$
M_{12}=K_{A} \Delta \varphi_{23}+K_{V} \Delta \dot{\varphi}_{23} .
$$

In this expression, the coefficients $K_{A}=J_{2}\left(\omega_{23}^{2}-\lambda_{1} \lambda_{2}\right)$ and $K_{V}=J_{2}\left(\lambda_{1}+\lambda_{2}\right)$ can be interpreted as new values of the stiffness and viscous friction coefficients in the oscillating system, which will provide the given law of change of the coordinate $\Delta \varphi_{23}$. The structural scheme of the three-mass oscillatory system, equipped with passive vibration protection of subsystem 1 in the form of an additional elastic element and viscous friction damper, is shown in Fig. 2.

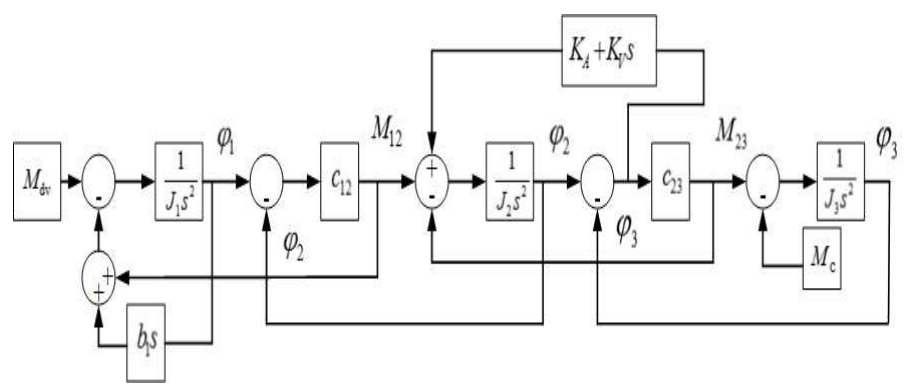

Fig. 2. Structural scheme of a passive vibration protection system

To find the law of change of the drive torque $M_{d v}$, let us consider subsystem 2 (see Fig. 1), the motion of subsystem 2 is described by the first and second equations of system (1): 


$$
\left\{\begin{array}{l}
J_{1} \ddot{\varphi}_{1}=M_{d v}-c_{12}\left(\varphi_{1}-\varphi_{2}\right)-b_{1} \dot{\varphi}_{1} \\
J_{2} \ddot{\varphi}_{2}=c_{12}\left(\varphi_{1}-\varphi_{2}\right)-c_{23}\left(\varphi_{2}-\varphi_{3}\right)
\end{array} .\right.
$$

Solving equations (6) relative to the elastic coordinate $\Delta \varphi_{12}$, we obtain:

$$
\Delta \ddot{\varphi}_{12}+\omega_{12}^{2} \Delta \varphi_{12}=M_{d v} / J_{1}+M_{23} / J_{2}-\frac{b_{1}}{J_{1}} \dot{\varphi}_{1}
$$

Here $\omega_{12}=\sqrt{\frac{c_{12}\left(J_{1}+J_{2}\right)}{J_{1} J_{2}}}$ - the partial oscillation frequency of subsystem 2. Taking into account that $M_{12}=c_{12} \Delta \varphi_{12}$, we determine from (4) the law of change of the elastic coordinate $\Delta \varphi_{12}$ :

$$
\Delta \varphi_{12}=\frac{J_{2}\left(\lambda_{1}^{2}+\omega_{23}^{2}\right)}{c_{12}} C_{1} e^{\lambda_{1} t}+\frac{J_{2}\left(\lambda_{2}^{2}+\omega_{23}^{2}\right)}{c_{12}} C_{2} e^{\lambda_{2} t} .
$$

Differentiating the expression (8) twice in time, we substitute the coordinate $\Delta \varphi_{12}$ and its second derivative into equation (7). We give the exponential law of the change of the moment transmitted through the elastic element with the stiffness coefficient $c_{23}$ in the form:

$$
M_{23}=c_{23}\left(C_{1} e^{\lambda_{1} t}+C_{2} e^{\lambda_{2} t}\right) .
$$

After some transformations, we find from expression (7) the required law of change of the driving torque:

$\left[\left(\lambda_{1}^{2}+\omega_{2}^{2}\right) K_{1}-\frac{c_{23}}{J_{2}}\right] C_{1} e^{\lambda_{1} t}+\left[\left(\lambda_{2}^{2}+\omega_{2}^{2}\right) K_{2}-\frac{c_{23}}{J_{2}}\right] C_{2} e^{\lambda_{2} t}=\frac{M_{d v}}{J_{1}}-\frac{b_{1}}{J_{1}} \dot{\varphi}_{1}$ (9)

where $K_{1}=\frac{J_{2}\left(\lambda_{1}^{2}+\omega_{23}^{2}\right)}{c_{12}}$ and $K_{2}=\frac{J_{2}\left(\lambda_{2}^{2}+\omega_{23}^{2}\right)}{c_{12}}$.

The found control law (9) is a function of time that does not depend on the structure of the controllers and provides the possibility of solving the problem of not only parametric, but also structural synthesis of the motion control system. To implement motion control on the principle of feedback, we express the time dependences (9) through the phase coordinates of the system. Substituting the coordinate $\Delta \varphi_{12}$ and its second derivative in (8), taking into account the initial conditions $\Delta \varphi_{12}(0)=\Delta \varphi_{12}$ and $\Delta \dot{\varphi}_{12}(0)=\Delta \dot{\varphi}_{12}$, we define the values of the exponential functions:

$C_{1} e^{\lambda_{1} t}=\frac{\lambda_{2} \Delta \varphi_{12}-\Delta \dot{\varphi}_{12}}{K_{1}\left(\lambda_{2}-\lambda_{1}\right)}$ (10) и $C_{2} e^{\lambda_{2} t}=\frac{-\lambda_{1} \Delta \varphi_{12}+\Delta \dot{\varphi}_{12}}{K_{2}\left(\lambda_{2}-\lambda_{1}\right)}$.

Substituting expressions (10) and (11) in (9) and grouping the coefficients of $\Delta \varphi_{12}$ and $\Delta \dot{\varphi}_{12}$, we obtain the dependence for determining the drive torque $M_{d v}$ :

$$
K_{V 1} \Delta \dot{\varphi}_{12}+K_{A 1} \Delta \varphi_{12}=\frac{M_{d v}}{J_{1}}-\frac{b_{1}}{J_{1}} \dot{\varphi}_{1}
$$

where:

$$
\begin{array}{r}
K_{A 1}=\frac{A_{1} \lambda_{2}}{K_{1}\left(\lambda_{2}-\lambda_{1}\right)}-\frac{A_{2} \lambda_{1}}{K_{2}\left(\lambda_{2}-\lambda_{1}\right)} ; \\
A_{1}=\left(\lambda_{1}^{2}+\omega_{12}^{2}\right) K_{1}-\frac{c_{12}}{J_{2}} ;
\end{array}
$$

$K_{V 1}=\frac{A_{2}}{K_{2}\left(\lambda_{2}-\lambda_{1}\right)}-\frac{A_{1}}{K_{1}\left(\lambda_{2}-\lambda_{1}\right)}$

$A_{2}=\left(\lambda_{2}^{2}+\omega_{12}^{2}\right) K_{1}-\frac{c_{12}}{J_{2}}$.

The coefficients $K_{A 1}$ and $K_{V 1}$ in expression (12) can be represented as the coefficients of additional feedbacks on the elastic coordinate $\Delta \varphi_{12}$ and its derivative. The structural scheme of the active oscillation control system based on additional feedbacks is shown in Fig. 3.

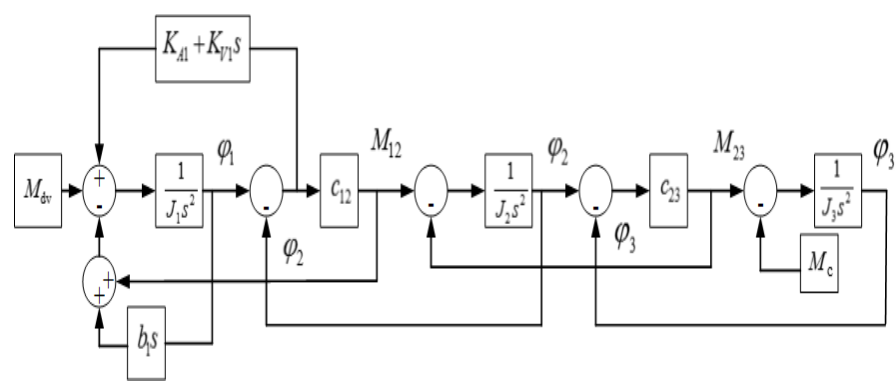

Fig. 3. Structural scheme of active oscillation control system

The unknowns in (12) are the coefficients $\lambda_{1}$ and $\lambda_{2}$. Determination of the values of these coefficients will be performed using the transfer function of the subsystem 1, provided that $M_{c}=0$ :

$$
W(p)=\frac{M_{23}}{M_{12}}=\frac{J_{3} c_{23}}{-J_{2} J_{3} s^{2}+J_{3} K_{V} s+\left(J_{3} K_{A}-c_{23}\left(J_{2}+J_{3}\right)\right)}
$$

From (13), revealing the coefficients of feedbacks, we obtain the characteristic equation of the system:

$$
s^{2}-\left(\lambda_{1}+\lambda_{2}\right) s+\lambda_{1} \lambda_{2}=0 .
$$

It is known that for a two-mass oscillating system the differential equation of the second order is determined by the expression:

$$
s^{2}+a_{1} s+a_{0}=0,
$$

where $a_{1}=2 \xi \omega_{0} ; \quad a_{0}=\left(\omega_{0}\right)^{2}, \quad \omega_{0}$ - frequency of oscillations; $\xi$ - damping coefficient; $s=d / d t$ [14].

Equating the coefficients of the corresponding powers in (14) and (15), we obtain the following relations:

$$
2 \xi \omega_{0}=-\lambda_{1}-\lambda_{2} ;\left(\omega_{0}\right)^{2}=\lambda_{1} \lambda_{2},
$$

of which we shall have:

$$
\lambda^{2}+\lambda_{2}^{2}-4 \xi^{2} \lambda_{1} \lambda_{2}=0 .
$$


A sufficient condition ensuring aperiodic transient processes in a two-mass system is the value of the damping coefficient $\xi \geq 0,707$, which corresponds to obtaining multiple roots of system, in this connection, the rigid feedback must be negative, and therefore $\lambda_{1} \lambda_{2}>\omega^{2}{ }_{12}$.

\section{RESEARCH AND DISCUSSION}

To test the effectiveness of the synthesized control of a three-mass oscillating system, numerical simulation of transient processes was carried out with the following parameters of the mechanical part: $J_{1}=4 \mathrm{~kg} \cdot \mathrm{m}^{2}$, $J_{2}=6 \mathrm{~kg} \cdot \mathrm{m}^{2}, \quad J_{3}=65 \mathrm{~kg} \cdot \mathrm{m}^{2}, \quad c_{12}=8000 \mathrm{~N} / \mathrm{m} / \mathrm{rad}$, $c_{12}=2000 \mathrm{~N} / \mathrm{m} / \mathrm{rad}, \quad b_{1}=15 \mathrm{~N} \cdot \mathrm{m} / \mathrm{sec} \quad$ [13]. For the specified parameters, the nearest multiple root $\lambda_{1}=\lambda_{2}=-20$; with necessary conditions $\lambda_{1} \neq \lambda_{2}$, we will take $\lambda_{2}=-25$ and $\lambda_{1}=-20$.

For the parameters adopted, the stiffness and viscous friction coefficients of the passive vibration protection system shown in Fig. 2, will have the values $K_{A}=-816 \mathrm{~N} / \mathrm{m}$ and $K_{V}=-270 \mathrm{~N} \cdot \mathrm{m} / \mathrm{s}$, respectively, and expression (12) will take the form:

$$
-71 \Delta \dot{\varphi}_{12}+1759 \Delta \varphi_{12}=\frac{M_{d v}}{J_{1}}-\frac{b_{1}}{J_{1}} \dot{\varphi}_{1} .
$$

During the simulation, the load torque in the first $M_{12}$ and second $M_{23}$ elastic elements, the speed of the third mass $\omega_{3}$, and the absolute deviations of this mass $\Delta \varphi_{13}=\varphi_{1}-\varphi_{3}$ in the start-up and load-step modes were determined. As an input, a step single signal of the drive torque $M_{d v}=1(t)$ passed through the delay link $\frac{1}{0.05 s+1}$ was used.

Executed studies have shown that the use of the passive vibration protection system (see Fig. 2) and the active control system (see Fig. 3) allows limiting the oscillational movements and providing aperiodic transients in the modes of start-up and load-step. As an illustration, Fig. 4 and 5 show the oscillogramms $M_{12}$ and $M_{23}, \omega_{3}$ and $\Delta \varphi_{13}$ for the start-up mode, and in Fig. 6 and 7 are the same oscillogramms obtained when the load is up on the actuating mechanism. the characteristic equation (14). As you know, positive feedbacks reduce the stability margin of the control
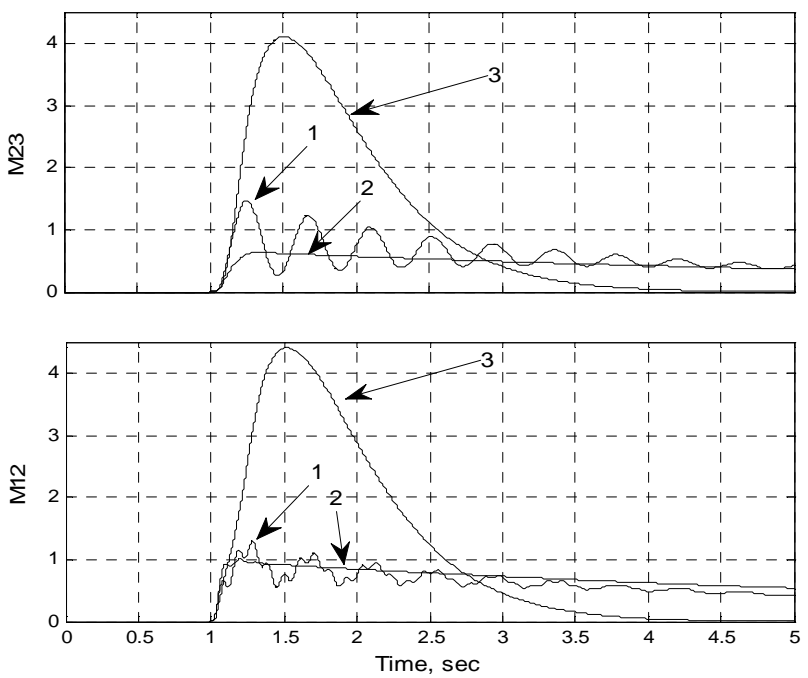

Fig. 4. Torque oscillogramms $M_{23}$ and $M_{12}$ in start-up mode
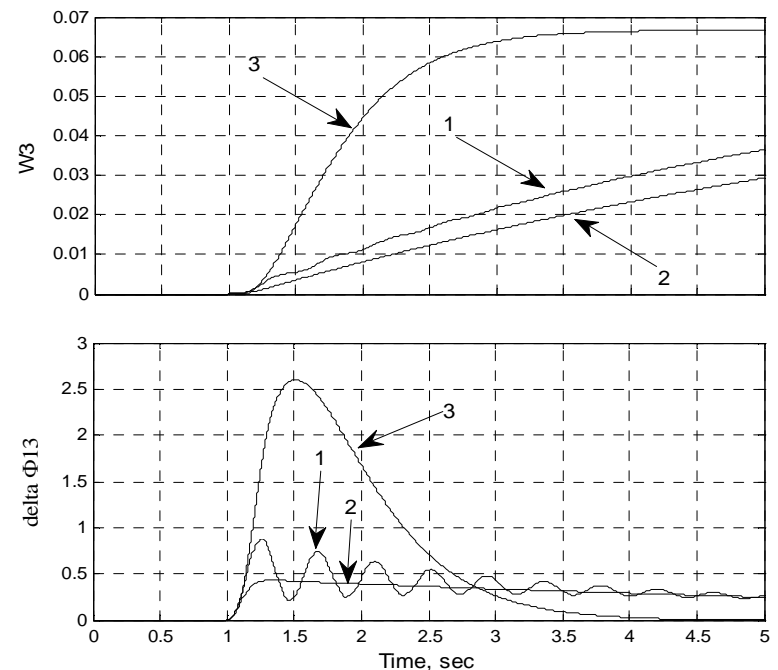

Fig. 5. Speed oscillogramms $\omega_{3}$ in start-up mode

In these figures curve 1 describes the transient processes occurring in the initial oscillatory system; curve 2 - processes in a system equipped with passive vibration protection unit, and curve 3 - processes in a system equipped with additional feedbacks for controlling oscillations. 

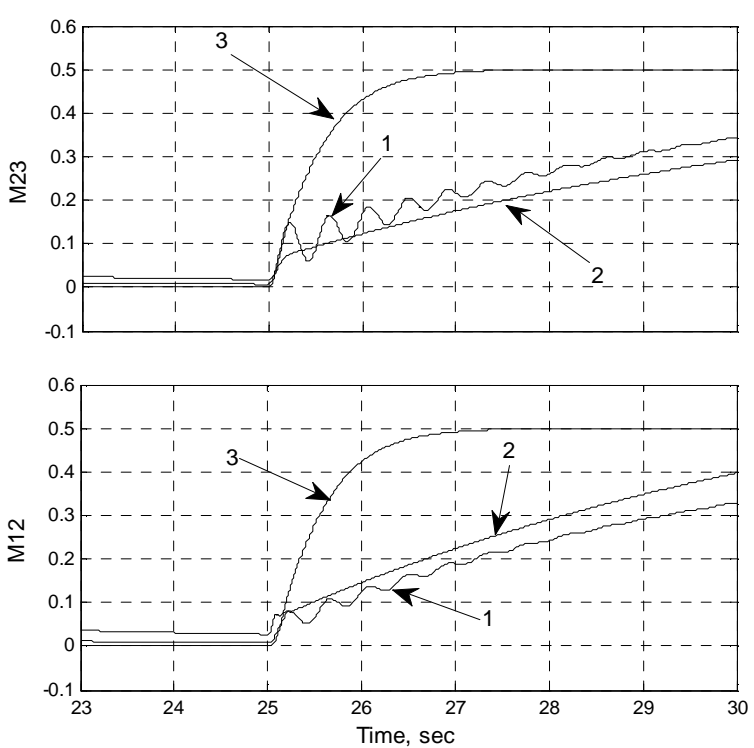

Fig. 6. Oscillogramms of elastic torques $M_{23}$ and $M_{12}$ in the load-step mode
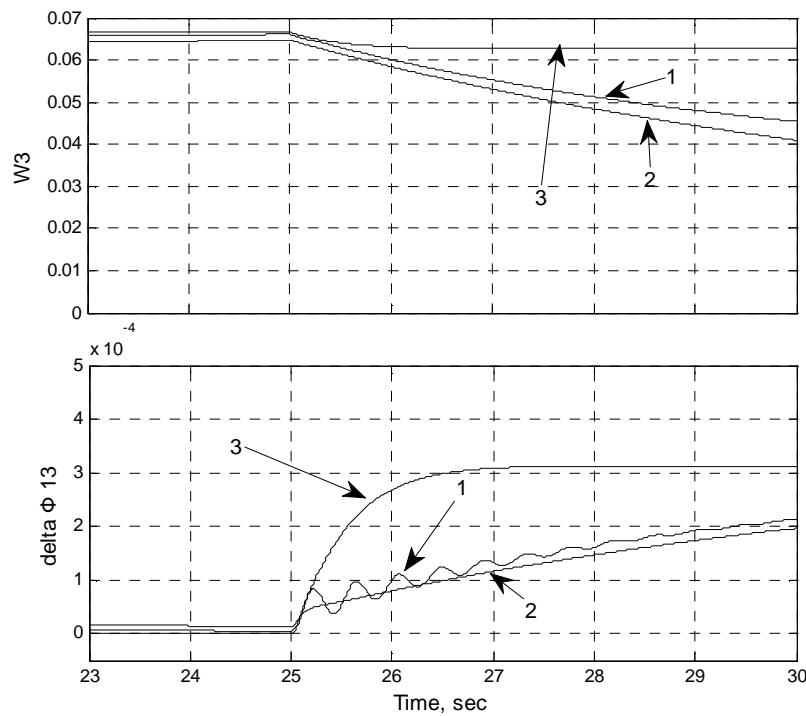

Fig. 7. Oscillogramms of speed $\omega_{3}$ and absolute deviation $\Delta \varphi_{13}$ in the load-step mode

As can be seen from the oscillogramms, the passive vibration protection system (curve 2) almost completely eliminates the elastic oscillations in the first partial system and ensures a sufficiently rapid damping of the oscillations in the second in the start-up mode (see Fig. 4) and the load-step in comparison with the initial oscillating system (see Fig. 6). The amplitude of the oscillations of the elastic torque $M_{23}$ is reduced by $60 \%$, and the torque $M_{12}$ by $22 \%$ compared to the initial system (curve 1). In this case, the deviation in the motion between the first and third masses $\Delta \varphi_{13}$ is monotonous in the start-up (see Fig. 5) and the load-step modes (see Fig. 7). However, in this case, a slight increase of the time of acceleration of the third mass (curve 2) was observed in comparison with the initial system (curve 1) (see Fig. 5 and 7).

An active oscillation control system based on additional feedbacks also provides a monotonous character of the change in the elastic moments (curve 3 ) with a transient time of about $2 \mathrm{sec}$ (see Fig. 4). However, significant amplitude ejections of these torques were observed in the start-up mode: the maximum value of the amplitude $M_{23}$ was 2.7 times, and $M_{12}$ was 3.3 times higher than in the original mechanical system (curve 1). In the load-step mode, elastic oscillation in partial systems are absent, and the torque vary according to an aperiodic law (see Fig. 6). It follows from Fig. 5 and 7, the new steady-state value of the third mass velocity $\omega_{3}$ (curve 3 ) is achieved rather quickly. The deviation in motion between the first and third masses in the starting mode (see Figure 5) is also monotonic with a large amplitude burst (curve 3 ), but in the load-step mode (see Fig. 7) this burst is not observed, and the magnitude deviation is determined by the load.

It should be noted that the passive vibration protection system ensures effective compensation of elastic oscillation only for the specified parameters of the protection object. The active oscillation control system based on additional feedbacks has an invariance to changes in the parameters of the oscillating system, since the feedback coefficients are determined directly by the mathematical model of the control object.

\section{CONCLUSION}

The method of synthesis of control actions proposed in this article, based on the determination by the solution of the inverse dynamic problems of control actions for individual partial systems, makes it possible to provide the desired character of the oscillating movements of the control object. Since the control actions obtained will be functions of time that do not impose any restrictions on the structure of the controllers, they can be realized by selecting the design parameters of the control object and using closed automatic control systems, which allows solving the problem of not only structural, but also parametric synthesis of motion control systems. Since the parameters of the control systems are determined directly from the mathematical model of the control object, the control obtained will have adaptive properties and weak sensitivity to parametric and coordinate perturbations. As shown by the conducted studies, the synthesized control allows reducing the level of elastic oscillations and dynamic loads in a three-mass oscillating system.

The proposed synthesis method can be used to control the movement not only of mining machines, but other technological and transport machines equipped with modern electric drive, the design schemes of which can be represented by three-mass oscillating systems. Its application will reduce the amplitude of elastic oscillation in transient modes and increase the efficiency, reliability and safety of such machines. 


\section{References}

[1] A.E. Kozyaruk, S.I. Taranov, A.V. Samolazov, «The direction of operation effectiveness encrease of excavating and automobile complexes on open-pit mining», Mining equipment and electromechanics, vol. 1, pp 6-11, 2014.

[2] «P\&H 2800XPC Electric mining shovel - AC Drive. Product overview», Joy Global Inc, 2012.

[3] S.I. Malafeev, A.A. Novgorodov, N.A. Serebrennikov, «New excavator EKG-18R: system of direct current motor drive with the pulse-width modulation control», Mining equipment and electromechanics, vol. 6, pp 21-25, 2012.

[4] N.K. Kuznetsov, D.E. Makhno,I.A. Iov, «Damping elastic oscillations of digging mechanism», IOP Conference Series: Earth and Environmental Science, vol. 87(2), paper 022011, October, 2017 [Conf. Innovations and Prospects of Development of Mining Machinery and Electrical Engeneering, March, vol. 87, 2017].

[5] A.C. Khatagov, A.M. Soin, Z.A. Khatagov, «Evaluation of the modernization efficiency of the rotation drive of mine shovel», Mining information and analytical bulletin, vol. 11, pp 80-85, 2008

[6] S.R. Ilin, S.S. Ilina, V.I. Samusya, Mechanic of hoists system, Dnepropetrovsk: Dnepropetrovsk National Mining University, 2014.
[7] A.G. Stepanov, M.V. Kornyakov, Machine Dynamics, Irkutsk: Irkutsk State Technical University, 2014.

[8] A.S. Leonenko, Methods and technical solution increase efficienci mine mashine in the North Area, Irkutsk: Irkutsk State Technical University, 2010.

[9] A.V. Lyakhomskiy, V.N. Fashilenko, Control of electromechanical mining machine systems, Moscow: Moscow State Mining University, 2004.

[10] G. Ya. Pyatibratov, «Conditions of optimization and efficiency of damping of oscillations of elastic mechanisms by an electric drive», Russian Electrical Engineering, vol. 86(7), pp 373-378, 2015.

[11] A.M. Samoylenko, «Programming control system tension lifting and traction cable dragline», Mining information and analytical bulletin, vol. 6, pp 476-479, 2011.

[12] P.D. Krut'ko, Inverse dynamics problems of control systems: Linear models, Moscow: Science, 1987.

[13] N.K. Kuznetsov, Dynamics of controlled machines with additional constraints, Irkutsk: Irkutsk State Technical University, 2009.

[14] R. Dorf, R. Bishop, Modern control systems, Eighth Edition, AddisonWesley, 2002. 